\title{
Inductive Limit Automorphisms of the Irrational Rotation Algebra
}

\author{
Samuel G. Walters \\ Department of Mathematics and Computer Science, The University of Northern British Columbia, \\ Prince George, British Columbia, V2N 4Z9 Canada. E-mail address: walters@unbc.edu
}

Received: 15 October 1993/in revised form: 11 November 1994

\section{Dedicated to Professor George Elliott on the occasion of his fiftieth birthday}

\begin{abstract}
It is shown that the flip automorphism $U \mapsto U^{*}, V \mapsto V^{*}$ of the irrational rotation algebra $\mathrm{A}_{0}$ is an inductive limit automorphism. Here, the algebra $\mathrm{A}_{\theta}$ is generated by unitaries $U, V$ satisfying $V U=\mathrm{e}^{2 \pi i \theta} U V$, where $\theta$ is an irrational number. Recently, Elliott and Evans proved that $\mathrm{A}_{\theta}$ can be approximated by unital subalgebras isomorphic to a direct sum of two matrix algebras over $C(\mathbb{T})$, the algebra of continuous functions on the unit circle. This is the central result which they used to obtain their structure theorem on $\mathrm{A}_{\theta}$; namely, that $\mathrm{A}_{\theta}$ is the inductive limit of an increasing sequence of subalgebras each isomorphic to a direct sum of two matrix algebras over $C(\mathbb{T})$. In their proof, they devised a subtle construction of two complementary towers of projections. In the present paper it is shown that the two towers can be chosen so that each summand of their approximating basic building blocks is invariant under the flip automorphism and, in particular, that the unit projection of the first summand is unitarily equivalent to the complement of the unit of the second by a unitary which is fixed under the flip. Also, an explicit computation of the flip on the approximating basic building blocks of $\mathrm{A}_{\theta}$ is given. Further, combining this result along with others, including a theorem of $\mathrm{Su}$ and a spectral argument of Bratteli, Evans, and Kishimoto, a two-tower proof is obtained of the fact established by Bratteli and Kishimoto that the fixed point subalgebra $\mathrm{B}^{\theta}$ (under the flip) is approximately finite dimensional. Also used here is the fact that $\mathrm{B}^{\theta}$ has the cancellation property and is gifted with four basic unbounded trace functionals. The question is raised whether other finite order automorphisms of $\mathrm{A}_{\theta}$ (arising from a matrix in $\operatorname{SL}(2, \mathbb{Z})$ ) are inductive limit automorphisms - or even almost inductive limit automorphisms in the sense of Voiculescu.
\end{abstract}

\section{Introduction}

In a recent paper, Elliott and Evans [6] devised a subtle construction of two towers of projections in their remarkable proof of the structure theorem on the irrational rotation algebra $A_{\theta}$. This result states that $A_{\theta}$ is the inductive limit of a sequence of direct sums of two matrix algebras over $C(\mathbb{T})$, the algebra of continuous functions 
over the unit circle $\mathbb{T}$. The objective of the present paper is two-fold. The main theorem is that the towers can be suitably chosen so that each of the two matrix algebra summands - see Theorem 1 of [6] - is invariant under the flip automorphism (see Sect. 2). The second objective is to combine this result along with others, including Su's classification theorem ([9] and [10]) and a spectral argument of Bratteli, Evans, and Kishimoto [2, Sect. 2], to give a two-tower proof that the fixed point subalgebra of $A_{\theta}$ under the flip automorphism is an AF-algebra (Sect. 3). The latter result was first proved by Bratteli and Kishimoto [4].

Let $\theta$ be an irrational number and let $\mathrm{A}_{\theta}$ denote the universal $\mathrm{C}^{*}$-algebra generated by two unitaries $U$ and $V$ such that $V U=\lambda U V$, where $\lambda=\mathrm{e}^{2 \pi i \theta}$. Recall that its canonical smooth dense subalgebra is

$$
\mathrm{A}_{\theta}^{\infty}=\left\{\sum_{m, n=-\infty}^{\infty} a_{m n} U^{m} V^{n}:\left\{a_{m n}\right\} \text { is rapidly decreasing }\right\},
$$

consisting of the smooth vectors in $\mathrm{A}_{\theta}$ under the canonical action of the 2-torus $\mathbb{T}^{2}$. For a given pair $(z, w) \in \mathbb{T}^{2}$, the canonical automorphism $\alpha_{z, w}$ satisfies $\alpha_{z, w}(U)=z U$ and $\alpha_{z, w}(V)=w V$. The subalgebra $\mathrm{A}_{\theta}^{\infty}$ is closed under the holomorphic functional calculus.

Let $\Phi$ denote the flip automorphism on $\mathrm{A}_{\theta}$ defined by $\Phi(U)=U^{*}, \Phi(V)=V^{*}$, and let $\mathrm{B}^{\theta}$ denote the fixed point subalgebra of $\mathrm{A}_{\theta}$ under $\Phi$, commonly called the non-commutative sphere. It is known that $\mathrm{B}^{\theta}$ is simple [3, p. 161] and has a unique tracial state [3, Theorem 4.5], denoted by $\tau$, given by the restriction of the trace state on $\mathrm{A}_{\theta}$. (A short proof of the uniqueness of trace is given in the appendix below). Similarly, the canonical smooth dense subalgebra of $\mathrm{B}^{\theta}$ is $\mathrm{B}^{\theta} \cap \mathrm{A}_{\theta}^{\infty}$ and consists of sums $\sum_{m, n} a_{m n} U^{m} V^{n}$, where $\left\{a_{m n}\right\}$ is rapidly decreasing and $a_{-m,-n}=a_{m n}$.

An interesting feature of the algebra $\mathrm{B}^{\theta}$ is that it possesses four basic unbounded trace functionals. They were first discovered by Bratteli, Elliott, Evans, and Kishimoto [3] and later (independently) by the author. Using the author's notation in [12], these trace functionals can be written as

$$
\phi_{i j}(a)=\sum_{m, n=-\infty}^{\infty} \lambda^{-(2 m-i)(2 n-j) / 2} a_{2 m-i, 2 n-j},
$$

for $i, j=0,1$, where $a=\sum a_{m n} U^{m} V^{n}$ and $\left\{a_{m n}\right\}$ is rapidly decreasing. For generic vectors $U^{m} V^{n}$,

$$
\phi_{i j}\left(U^{m} V^{n}\right)=\lambda^{-m n / 2} \delta_{i, \bar{m}} \delta_{j, \bar{n}},
$$

for $m, n \in \mathbb{Z}$, where $\bar{m}$ is $m$ reduced modulo 2 (so $\bar{m}=0,1$ ) and $\delta_{i j}$ is the Kronecker $\delta$-function. For smooth vectors $a, b \in \mathrm{A}_{\theta}, \phi_{l j}$ satisfies the following "twisted" tracial property with respect to the flip automorphism:

$$
\phi_{\imath j}(a b)=\phi_{i j}(\Phi(b) a) .
$$

It follows that $\phi_{i j}$ is tracial on $\mathrm{B}^{\theta}$.

Putting these trace functionals together along with the (bounded) trace $\tau$, introduce the (densely defined) vector trace $\vec{\tau}$ on the fixed point algebra $\mathrm{B}^{\theta}$ by setting

$$
\vec{\tau}(x)=\left(\tau(x) ; \phi_{00}(x), \phi_{01}(x), \phi_{10}(x), \phi_{11}(x)\right),
$$


for (smooth) $x \in \mathrm{B}^{\theta}$. It is known that $\vec{\tau}$ induces an injective map on $K_{0}\left(\mathrm{~B}^{\theta}\right)$ (with values in $\mathbb{R}^{5}$ ) and that for any two projections $p, q \in M_{k}\left(\mathrm{~B}^{\theta}\right): \vec{\tau}[p]=\vec{\tau}[q]$ if and only in $p$ and $q$ are unitarily equivalent in $M_{k}\left(\mathrm{~B}^{\theta}\right)$ [12, Corollary 5.6]. If $p$ and $q$ are smooth projections the unitary implementing the equivalence can be chosen to be smooth. This fact will be used in the proofs below. Note that the vector trace of the identity element $1 \in \mathrm{B}^{\theta}$ is $\vec{\tau}(1)=(1 ; 1,0,0,0)$.

Remark. In [12] the vector trace was actually considered on the crossed product $\mathrm{A}_{\theta} \times{ }_{\Phi} \mathbb{Z}_{2}$ and computed on its $K_{0}$-group. But this algebra is strongly Morita equivalent to the fixed point algebra $\mathrm{B}^{\theta}$ and so justifies the above application of [12, Corollary 5.6].

As in [6], recall that a tower of projections in $\mathrm{A}_{\theta}$ is defined by a projection $\mathbf{e}$ in $\mathrm{A}_{\theta}$ and the (canonical) action of some cyclic subgroup $G$ of $\mathbb{T}^{2}$, with generator $t \in \mathbb{T}^{2}$, such that $\mathbf{e}, \alpha_{t}(\mathbf{e}), \ldots, \alpha_{t}^{q-1}(\mathbf{e})$ are mutually orthogonal projections. Here, $q$ is the order of $G$ and is called the height of the tower. The projection $\mathbf{e}$ will sometimes be referred to as the base projection of the tower. The unit of the tower is the sum $\sum_{j} \alpha_{t}^{j}(\mathbf{e})$ of its projections. Clearly, the trace of $\mathbf{e}$ is at most $\frac{1}{q}$.

Some interesting questions arise about certain automorphisms of the irrational rotation algebra.

Questions and Comments. Let $\sigma$ be an automorphism of $\mathrm{A}_{\theta}$ arising from a matrix in $\operatorname{SL}(2, \mathbb{Z})$ (which has a natural action on $A_{\theta}$ in the sense of Brenken and Watatani). Is every finite-order $\sigma$ an inductive limit automorphism (with respect to some choice of the basic building blocks of Elliott and Evans)? Does the fixed point subalgebra $\mathrm{A}_{\theta}^{\sigma}$ have real rank zero (cf. Theorem 3.0 below)? Does it have the cancellation property? If $K_{1}\left(\mathrm{~A}_{\theta}^{\sigma}\right)=0$, is $\mathrm{A}_{\theta}^{\sigma}$ an AF-algebra? Does the fixed point algebra have an ample collection of unbounded trace functionals? If $\sigma$ is not an inductive limit automorphism is it (at least) an almost inductive limit automorphism in the sense of Voiculescu [11] with respect to the basic building blocks of Elliott-Evans?

These questions are unknown for the order 4 automorphism of $\mathrm{A}_{\theta}$ defined by $U \mapsto V, V \mapsto U^{*}$. Except that the $K_{0}$-group of its corresponding fixed point subalgebra $\mathrm{B}$ is probably $\mathbb{Z}^{9}$ (G. Elliott, private communication). We know that $\mathrm{B}$ has lots of projections, but probably not of Rieffel type. This follows from earlier work of George Elliott on the gaps in the spectrum of the almost Mathieu operator $H=U+V+U^{*}+V^{*}$ (at least for irrationals close enough to $\frac{1}{3}$, since $H$ is in the fixed point algebra B). Perhaps the spectral projections of $H$ can be used as model projections in a new tower construction for this situation.

\section{The Main Result}

The first objective of this section is to prove that for any

$$
\left[\begin{array}{ll}
p^{\prime} & p \\
q^{\prime} & q
\end{array}\right] \in \mathrm{SL}(2, \mathbb{Z})
$$

with $q, q^{\prime}>0$ and $\frac{p}{q}<\theta<\frac{p^{\prime}}{q^{\prime}}$, the two towers constructed in [6] of heights $q$ and $q^{\prime}$ with base projections (in $\mathrm{A}_{\theta}$ ) having traces $\beta=p^{\prime}-q^{\prime} \theta$ and $\beta^{\prime}=-p+q \theta$, respectively, can be chosen so that their units are in $\mathrm{B}^{\theta}$ and, more importantly, that 
the unit of the second tower is unitarily equivalent in $\mathrm{B}^{\theta}$ to the complement of the unit of the first tower. The second objective is to show that these towers give rise to basic building blocks of $\mathrm{A}_{\theta}$ which are invariant under the flip automorphism.

Note that $q \beta+q^{\prime} \beta^{\prime}=1$ so that

$$
\beta<\frac{1}{q} \quad \text { and } \quad \beta^{\prime}<\frac{1}{q^{\prime}} .
$$

The base projection of the first tower will be taken to be the Rieffel projection (as in $[6$, p. 480$]$ )

$$
\mathbf{e}_{\beta}=V^{-q^{\prime}} g_{1}(U)+f_{1}(U)+g_{1}(U) V^{q^{\prime}}
$$

where $f_{1}$ is a smooth function which is supported in an interval of length $\frac{1}{q}$ (in $\mathbb{R} / \mathbb{Z}$ ) and has trace $\beta$. This projection generates a tower of height $q$ under the canonical action of the cyclic subgroup $\left\{z^{j}: 0 \leqq j<q\right\} \times\{1\}$ of $\mathbb{T} \times \mathbb{T}$, where $z=\mathrm{e}^{2 \pi l / q}$. The unit of this tower is easily seen to be the smooth projection

$$
E_{1}=V^{-q^{\prime}} G_{1}(U)+F_{1}(U)+G_{1}(U) V^{q^{\prime}},
$$

where

$$
F_{1}(U)=\sum_{j=0}^{q-1} f_{1}\left(z^{j} U\right) \quad \text { and } \quad G_{1}(U)=\sum_{j=0}^{q-1} g_{1}\left(z^{j} U\right) .
$$

The graphs of $F_{1}$ and $G_{1}$ are just the sum of all the shifts of the graphs of $f_{1}$ and $g_{1}$, respectively, by length all integer multiples of $\frac{1}{q}$ along $\mathbb{R} / \mathbb{Z}$. In order for $E_{1}$ to be in $\mathrm{B}^{\theta}$ the following conditions on $F_{1}$ and $G_{1}$ must be satisfied:

$$
F_{1}(-t)=F_{1}(t), \quad G_{1}(\beta-t)=G_{1}(t),
$$

for $t \in \mathbb{R} / \mathbb{Z}$, as can easily be checked.

The second tower is based on the Rieffel projection $([6, \mathrm{p} .481])$

$$
\mathbf{e}_{\beta^{\prime}}=U^{q} g_{2}(V)+f_{2}(V)+g_{2}(V) U^{-q},
$$

where $f_{2}$ is a smooth function which is supported in an interval of length $\frac{1}{q^{\prime}}$ and has trace $\beta^{\prime}$. This projection generates a tower of height $q^{\prime}$ under the canonical action of the cyclic subgroup $\{1\} \times\left\{w^{j}: 0 \leqq j<q^{\prime}\right\}$ of $\mathbb{T} \times \mathbb{T}$, where $w=\mathrm{e}^{2 \pi i / q^{\prime}}$. The unit of this tower is the smooth projection

$$
E_{2}=U^{q} G_{2}(V)+F_{2}(V)+G_{2}(V) U^{-q},
$$

where

$$
F_{2}(V)=\sum_{j=0}^{q^{\prime}-1} f_{2}\left(w^{j} V\right) \text { and } G_{2}(V)=\sum_{j=0}^{q^{\prime}-1} g_{2}\left(w^{j} V\right) .
$$

The graphs of $F_{2}$ and $G_{2}$ are just the sum of all the shifts of those of $f_{2}$ and $g_{2}$, respectively, by length all integer multiples of $\frac{1}{q^{\prime}}$ along the unit interval. Similarly, in order that $E_{2}$ be in $\mathrm{B}^{\theta}$ the following conditions on $F_{2}$ and $G_{2}$ are required:

$$
F_{2}(-t)=F_{2}(t), \quad G_{2}\left(-\beta^{\prime}-t\right)=G_{2}(t) .
$$


Now a specific choice of the functions $f_{1}, g_{1}, f_{2}, g_{2}$ will be given in each of the following two cases.

Case 1. $\beta>\frac{1}{2 q}$. Define a smooth function $f_{1}$ such that $f_{1}=1$ on $\left[\frac{1}{q}-\beta, \beta\right], f_{1}=0$ on $\left[\frac{1}{q}, 1\right]$, and $f_{1}(t)=1-f_{1}(t-\beta)$ for $t \in\left[\beta, \frac{1}{q}\right]$. Put $g_{1}=(-1)^{q} \sqrt{f_{1}-f_{1}^{2}}$ on $\left[\beta, \frac{1}{q}\right]$ and $g_{1}=0$ elsewhere. Inspection of the graphs of $F_{1}$ and $G_{1}$ shows that they satisfy $(\star 1)$ and a little arithmetic gives the values

$$
F_{1}(0)=0, \quad F_{1}\left(\frac{1}{2}\right)=\delta_{\bar{q}, 1}, \quad G_{1}\left(\frac{\beta}{2}-\frac{k}{2}\right)=\frac{1}{2}(-1)^{q} \delta_{\bar{k}, 1} \delta_{\bar{q}, 1},
$$

for all $k \in \mathbb{Z}$. The latter values of $G_{1}$ can be obtained by noting that it is supported on the union of the intervals $\left[\beta+\frac{j}{q}, \frac{j+1}{q}\right], j=0,1, \ldots, q-1$, and that $\frac{\beta}{2}-\frac{k}{2}(\bmod 1)$ is a midpoint of one such interval (where $G_{1}$ is $\frac{1}{2}(-1)^{q}$ ) if both $k$ and $q$ are odd, and otherwise $\frac{\beta}{2}-\frac{k}{2}(\bmod 1)$ is not in their union.

Since $\beta^{\prime}<\frac{1}{2 q^{\prime}}$, for the second tower choose a smooth function $f_{2}$ such that $f_{2}=1$ on $\left[0, \frac{1}{3} \beta^{\prime}\right] \cup\left[1-\frac{1}{3} \beta^{\prime}, 1\right], f_{2}=0$ on $\left[\frac{2}{3} \beta^{\prime}, 1-\frac{2}{3} \beta^{\prime}\right]$ and $f_{2}(t)=1-f_{2}\left(-\beta^{\prime}+\right.$ $t$ ) for $t \in\left[\frac{1}{3} \beta^{\prime}, \frac{2}{3} \beta^{\prime}\right]$. Let $g_{2}=\sqrt{f_{2}-f_{2}^{2}}$ on $\left[1-\frac{2}{3} \beta^{\prime}, 1-\frac{1}{3} \beta^{\prime}\right]$, and $g_{2}=0$ elsewhere. The graphs of $F_{2}$ and $G_{2}$ show that they satisfy $(\star 2)$ and a similar arithmetic gives the values

$$
F_{2}(0)=1, \quad F_{2}\left(\frac{1}{2}\right)=\delta_{q^{\prime}, 0}, \quad G_{2}\left(-\frac{\beta^{\prime}}{2}-\frac{k}{2}\right)=\frac{1}{2} \delta_{\overline{k q^{\prime}}, 0},
$$

for all $k \in \mathbb{Z}$.

It is important to recall that $f_{1}$ (and hence $F_{1}$ ) should be chosen so that its derivative is bounded (say, as in $\left[6\right.$, p. 485]) by $6 \max \left\{\beta^{-1},\left(\frac{1}{q}-\beta\right)^{-1}\right\}=$ $6\left(\frac{1}{q}-\beta\right)^{-1}$. This will also be required of $f_{2}$ (and hence $F_{2}$ ), where $\beta$ is replaced by $\beta^{\prime}$ and $q$ by $q^{\prime}$, as well as for the choices made in the next case. This is needed in order to control the estimates in [6].

Case 2. $\beta<\frac{1}{2 q}$. Let $f_{1}$ be a smooth function such that $f_{1}=1$ on $\left[0, \frac{1}{3} \beta\right] \cup$ $\left[1-\frac{\beta}{3}, 1\right], f_{1}=0$ on $\left[\frac{2}{3} \beta, 1-\frac{2}{3} \beta\right]$ and $f_{1}(t)=1-f_{1}(-\beta+t)$ for $t \in\left[\frac{1}{3} \beta, \frac{2}{3} \beta\right]$. Define $g_{1}=\sqrt{f_{1}-f_{1}^{2}}$ on $\left[\frac{1}{3} \beta, \frac{2}{3} \beta\right]$, and $g_{1}=0$ elsewhere. The graphs of $F_{1}$ and $G_{1}$ show that they satisfy $(\star 1)$ and have values

$$
F_{1}(0)=1, \quad F_{1}\left(\frac{1}{2}\right)=\delta_{\bar{q}, 0}, \quad G_{1}\left(\frac{\beta}{2}-\frac{k}{2}\right)=\frac{1}{2} \delta_{\overline{k q}, 0},
$$

for all $k \in \mathbb{Z}$, which are analogous to the values of $F_{2}$ and $G_{2}$ in case 1 .

Since $\beta^{\prime}>\frac{1}{2 q^{\prime}}$, for the second tower choose a smooth function $f_{2}$ such that $f_{2}=1$ on $\left[\frac{1}{q^{\prime}}-\beta^{\prime}, \beta^{\prime}\right], f_{2}=0$ on $\left[\frac{1}{q^{\prime}}, 1\right]$, and $f_{2}(t)=1-f_{2}\left(t-\beta^{\prime}\right)$ for $t \in\left[\beta^{\prime}, \frac{1}{q^{\prime}}\right]$. Put $g_{2}=(-1)^{q^{\prime}} \sqrt{f_{2}-f_{2}^{2}}$ on $\left[0, \frac{1}{q^{\prime}}-\beta^{\prime}\right]$ and $g_{2}=0$ elsewhere. Again, $F_{2}$ and $G_{2}$ satisfy $(\star 2)$ and have the values

$$
F_{2}(0)=0, \quad F_{2}\left(\frac{1}{2}\right)=\delta_{q^{\bar{\gamma}}, 1}, \quad G_{2}\left(-\frac{\beta^{\prime}}{2}-\frac{k}{2}\right)=\frac{1}{2}(-1)^{q^{\prime}} \delta_{\bar{k}, 1} \delta_{\bar{q}^{\bar{l}}, 1},
$$

for all $k \in \mathbb{Z}$, which are analogous to the values of $F_{1}$ and $G_{1}$ in case 1 . 
The next lemma gives the unbounded traces of the unit projections $E_{1}$ and $E_{2}$ in terms of point evaluations.

2.1. Lemma. For $i, j=0,1$ one has:

$$
\begin{aligned}
\phi_{i j}\left(E_{1}\right)= & \frac{\delta_{j, 0}}{2}\left(F_{1}(0)+(-1)^{i} F_{1}\left(\frac{1}{2}\right)\right) \\
& +\delta_{j, q^{\prime}}\left(G_{1}\left(\frac{\beta}{2}-\frac{p^{\prime}}{2}\right)+(-1)^{i} G_{1}\left(\frac{\beta}{2}-\frac{p^{\prime}}{2}+\frac{1}{2}\right)\right),
\end{aligned}
$$

and

$$
\begin{aligned}
\phi_{i j}\left(E_{2}\right)= & \frac{\delta_{i, 0}}{2}\left(F_{2}(0)+(-1)^{j} F_{2}\left(\frac{1}{2}\right)\right) \\
& +\delta_{l, \bar{q}}\left(G_{2}\left(-\frac{\beta^{\prime}}{2}-\frac{p}{2}\right)+(-1)^{j} G_{2}\left(-\frac{\beta^{\prime}}{2}-\frac{p}{2}+\frac{1}{2}\right)\right) .
\end{aligned}
$$

Proof. It is enough to prove the first equality, the second being similar. The computation is the same as that done in $[12$, Sect. 3] which is repeated here for the sake of completeness. Recall from above that $E_{1}$ is in $\mathrm{B}^{\theta}$ and, fixing $i$ and $j$, one has

$$
\begin{aligned}
\phi_{i j}\left(E_{1}\right) & =\phi_{i j}\left(V^{-q^{\prime}} G_{1}(U)+F_{1}(U)+G_{1}(U) V^{q^{\prime}}\right) \\
& =\phi_{i j}\left(F_{1}(U)\right)+2 \phi_{i j}\left(G_{1}(U) V^{q^{\prime}}\right)
\end{aligned}
$$

The quantities $\phi_{i j}\left(F_{1}(U)\right)$ and $\phi_{i j}\left(G_{1}(U) V^{q^{\prime}}\right)$ are worked out separately. First write the Fourier series of $F_{1}$ as $F_{1}(z)=\sum_{n} a_{n} z^{n}$, where $z \in \mathbb{T}$ and $\left\{a_{n}\right\}$ is rapidly decreasing. The above expression becomes

$$
\begin{aligned}
\phi_{i j}\left(F_{1}(U)\right) & =\sum_{n=-\infty}^{\infty} a_{n} \phi_{i j}\left(U^{n}\right) \\
& =\sum_{n=-\infty}^{\infty} a_{n} \delta_{i, n} \delta_{j, 0} \\
& =\delta_{j, 0} \sum_{k=-\infty}^{\infty} a_{i+2 k} .
\end{aligned}
$$

The last sum can be found by splitting the series for $F_{1}(1)$ and $F_{1}(-1)$ according to the indices $i+2 k$ and $i+2 k+1$ and thereby obtaining $\sum_{k} a_{i+2 k}=\frac{1}{2}\left(F_{1}(1)+\right.$ $\left.(-1)^{i} F_{1}(-1)\right)$. Hence

$$
\begin{aligned}
\phi_{i j}\left(F_{1}(U)\right) & =\frac{1}{2} \delta_{j, 0}\left(F_{1}(1)+(-1)^{i} F_{1}(-1)\right) \\
& =\frac{1}{2} \delta_{j, 0}\left(F_{1}(0)+(-1)^{i} F_{1}\left(\frac{1}{2}\right)\right),
\end{aligned}
$$


where in the last equality $F_{1}$ is written as a function of $t \in \mathbb{R} / \mathbb{Z}$. To find $\phi_{i j}\left(G_{1}(U) V^{q^{\prime}}\right)$, one similarly writes out the Fourier series of $G_{1}$ as $G_{1}(z)=\sum_{n} b_{n} z^{n}$ and obtains

$$
\begin{aligned}
\phi_{i j}\left(G_{1}(U) V^{q^{\prime}}\right) & =\sum_{n=-\infty}^{\infty} b_{n} \phi_{i j}\left(U^{n} V^{q^{\prime}}\right) \\
& =\sum_{n=-\infty}^{\infty} b_{n} \lambda^{-n q^{\prime} / 2} \delta_{l, \bar{n}} \delta_{j, q^{\prime}} \\
& =\delta_{j, q^{\prime}} \sum_{k=-\infty}^{\infty} b_{i+2 k} \lambda^{-(i+2 k) q^{\prime} / 2} \\
& =\delta_{j, q^{\prime}} \lambda^{-i q^{\prime} / 2} \sum_{k=-\infty}^{\infty} b_{i+2 k} \lambda^{-k q^{\prime}} .
\end{aligned}
$$

To determine the last sum one splits the series for $G_{1}$ as follows:

$$
G_{1}(z)=z^{l} \sum_{k=-\infty}^{\infty} b_{i+2 k} z^{2 k}+z^{1+i} \sum_{k=-\infty}^{\infty} b_{1+i+2 k} z^{2 k}
$$

Evaluation at $z=\lambda^{-q^{\prime} / 2}$ (or $t=\frac{\beta}{2}-\frac{p^{\prime}}{2}$ ) gives

$$
G_{1}\left(\frac{\beta}{2}-\frac{p^{\prime}}{2}\right)=\lambda^{-q^{\prime} i / 2} \sum_{k=-\infty}^{\infty} b_{i+2 k} \lambda^{-k q^{\prime}}+\lambda^{-q^{\prime}(i+1) / 2} \sum_{k=-\infty}^{\infty} b_{1+i+2 k} \lambda^{-k q^{\prime}},
$$

and at $z=-\lambda^{-q^{\prime} / 2}$ (or $t=\frac{\beta}{2}-\frac{p^{\prime}}{2}+\frac{1}{2}$ ) the series becomes

$$
\begin{aligned}
G_{1}\left(\frac{\beta}{2}-\frac{p^{\prime}}{2}+\frac{1}{2}\right)= & (-1)^{i} \lambda^{-q^{\prime} i / 2} \sum_{k=-\infty}^{\infty} b_{l+2 k} \lambda^{-k q^{\prime}} \\
& -(-1)^{i} \lambda^{-q^{\prime}(i+1) / 2} \sum_{k=-\infty}^{\infty} b_{1+i+2 k} \lambda^{-k q^{\prime}} .
\end{aligned}
$$

Multiplying this by $(-1)^{i}$ and adding the result to the previous term yields

$$
\phi_{i j}\left(G_{1}(U) V^{q^{\prime}}\right)=\frac{1}{2} \delta_{j, q^{\prime}}\left(G_{1}\left(\frac{\beta}{2}-\frac{p^{\prime}}{2}\right)+(-1)^{i} G_{1}\left(\frac{\beta}{2}-\frac{p^{\prime}}{2}+\frac{1}{2}\right)\right),
$$

which together with $(\star)$ gives the first equality of the lemma.

Inserting the values of $F_{1}, G_{1}, F_{2}$ and $G_{2}$ found in each of the above two cases into the unbounded trace expressions of Lemma 2.1 it can be checked, with a little arithmetic, that the following equality of vector traces holds

$$
\vec{\tau}\left(E_{1}\right)+\vec{\tau}\left(E_{2}\right)=\vec{\tau}(1) .
$$

(Here, 1 is the identity element of $\mathrm{B}^{\theta}$.) In fact, since cases 1 and 2 are dual to each other it is enough to check this for just one of them. Thus, $\vec{\tau}\left(E_{1}\right)=\vec{\tau}\left(1-E_{2}\right)$ and so $E_{1}$ is unitarily equivalent in $\mathrm{B}^{\theta}$ to $1-E_{2}$ (by [12, Corollary 5.6]), as was required above. Let $W$ be a smooth unitary in $\mathrm{B}^{\theta}$ such that $E_{1}=W\left(1-E_{2}\right) W^{*}$.

In their proof of the structure theorem, Elliott and Evans showed that the irrational rotation algebra $\mathrm{A}_{\theta}$ can be approximated by a sequence of (unital) subalgebras of the form $C_{q} \oplus D_{q^{\prime}}$ (orthogonal sum), where $C_{q} \cong M_{q}(C(\mathbb{T})$ ) and 
$D_{q^{\prime}} \cong M_{q^{\prime}}(C(\mathbb{T}))$ are the basic building blocks [6, Theorem 1]. Let us now digress and recall briefly their construction of the matrix units and generators of these subalgebras, as these are at the heart of the proof.

Since the first tower was generated by the projection $\mathbf{e}_{\beta}$, one lets

$$
\mathbf{e}_{11}=\mathbf{e}_{\beta} \quad \text { and } \quad \mathbf{e}_{22}=\alpha\left(\mathbf{e}_{\beta}\right),
$$

where $\alpha=\alpha_{z, 1}^{p}\left(z=\mathrm{e}^{2 \pi i / q}\right)$ and the projections $\mathbf{e}_{11}$ and $\mathbf{e}_{22}$ are mutually orthogonal. Next, $\left|\mathbf{e}_{22} V \mathbf{e}_{11}\right|$ is an invertible element of $\mathbf{e}_{11} \mathrm{~A}_{\theta} \mathbf{e}_{11}$ (where $|x|=\left(x^{*} x\right)^{\frac{1}{2}}$ ) and so the unique partial isometry, denoted by $\mathbf{e}_{21}$, of the polar decomposition of $\mathbf{e}_{22} V \mathbf{e}_{11}$ belongs to $A_{\theta}$. Then, as in $[6$, p. 490], put

$$
\mathbf{e}_{2+i, 2+i}=\alpha^{l}\left(\mathbf{e}_{22}\right), \quad \mathbf{e}_{2+i, 1+i}=\alpha^{i}\left(\mathbf{e}_{21}\right), \quad 0 \leqq i \leqq q-2,
$$

and let $\left(\mathbf{e}_{i j}\right)_{1 \leqq i, j \leqq q}$ be the completion of $(\star \star)$ to a system of $q \times q$ matrix units. Finally, there is a unitary $v$ in $\mathbf{e}_{11} \mathrm{~A}_{\theta} \mathbf{e}_{11}$ such that

$$
\alpha^{q-1}\left(\mathbf{e}_{21}\right)=v \mathbf{e}_{1 q} .
$$

The subalgebra $C_{q}$ is generated by the matrix units $\left(\mathbf{e}_{i j}\right)$ and $v$. Its identity is the unit of the first tower $\sum_{i} \mathbf{e}_{i i}=E_{1}$, which is fixed under the flip.

In the same way the second tower is generated by the projection $\mathbf{e}_{\beta^{\prime}}$ under the action of $\alpha^{\prime}=\alpha_{1, w}^{-p^{\prime}}$, where $w=\mathrm{e}^{2 \pi i / q^{\prime}}$. So, $\mathbf{e}_{11}^{\prime}=\mathbf{e}_{\beta^{\prime}}$ and $\mathbf{e}_{22}^{\prime}=\alpha^{\prime}\left(\mathbf{e}_{\beta^{\prime}}\right)$ and the rest of the construction proceeds along the same lines of the preceding paragraph giving the subalgebra $C_{q^{\prime}}^{\prime}$ generated by the matrix units $\left(\mathbf{e}_{i j}^{\prime}\right)$ and a unitary $u^{\prime}$ analogous to $v$ above (see $[6, \mathrm{p} .490]$ ). The identity element of $C_{q^{\prime}}^{\prime}$ is $\sum_{i} \mathbf{e}_{l i}^{\prime}=E_{2}$, which is also fixed under the flip.

Now one lets $D_{q^{\prime}}=W C_{q^{\prime}}^{\prime} W^{*}$ which has identity element $W E_{2} W^{*}=1-E_{1}$ so that the subalgebra $C_{q} \oplus D_{q^{\prime}}$ has the same identity of $\mathrm{A}_{\theta}$. As required by the construction of Elliott and Evans, it is important that this subalgebra contain elements which approximate the canonical generators $U$ and $V$ of $\mathrm{A}_{\theta}$. This follows because $W$ approximately commutes with $U$ and $V$ as shown by the following remark.

Technical Remark. The classes of $E_{1}$ and $1-E_{2}$ are equal in $K_{0}\left(\mathrm{~A}_{\theta}\right)$ and hence $E_{1}$ and $1-E_{2}$ are unitarily equivalent in $\mathrm{A}_{\theta}$ (by [8, Corollary 2.5]) - in fact, by a smooth unitary. Call this unitary $w$. It is important to point out that $w$ is chosen very carefully in [6]. Namely, the derivatives of $w$ under the canonical basis of derivations are required to be appropriately bounded in norm (see [6, p. 483-484]). This will guarantee that $w$ approximately commutes with $U$ and $V$. However, a careful examination of the proof of Theorem 1 in [6] will show that the bound on the canonical derivatives of $w$ is determined by the bound on the derivatives of the functions $F_{1}$ and $F_{2}$ corresponding to the units $E_{1}$ and $E_{2}$, respectively. Thus the bound on the derivatives of $W$ is determined by the bound on the derivatives of the functions $F_{1}$ and $F_{2}$, which is no more than, say, 3 times the bounds in [6] as remarked at the end of case 1 above. It follows that the remainder of the proof of [6] remains unchanged and so $W$ will have appropriate bounds on the norm of its derivatives.

In showing that the flip automorphism is an inductive limit automorphism, both $C_{q}$ and $D_{q^{\prime}}$ must be invariant under the flip. This is shown here only for $C_{q}$, the algebra $C_{q^{\prime}}^{\prime}$ being similarly invariant and hence so is $D_{q^{\prime}}=W C_{q^{\prime}}^{\prime} W^{*}$ since $W$ is 
fixed under the flip. To show that $C_{q}$ is invariant, use will be made of the following two lemmas, which will also be required in the next section.

For arbitrary integers $m, n$ it will be convenient to write $\mathbf{e}_{m n}=\mathbf{e}_{m^{\prime} n^{\prime}}$, where $m^{\prime} \equiv m(\bmod q), n^{\prime} \equiv n(\bmod q)$ and $1 \leqq m^{\prime}, n^{\prime} \leqq q$.

Notation. Throughout the rest of the paper, $r$ will denote the unique positive integer such that $r \equiv 1-q^{\prime}(\bmod q)$ and $1 \leqq r \leqq q$. This will be relevant only for the case $\beta>\frac{1}{2 q}$.

The following lemma shows that the flip automorphism actually flips each of the towers.

\subsection{Lemma.}

(i) If $\beta>\frac{1}{2 q}$, then $\Phi\left(\mathbf{e}_{k+1, k+1}\right)=\mathbf{e}_{r-k, r-k}$, for $k=0,1, \ldots, q-1$.

(ii) If $\beta<\frac{1}{2 q}$, then $\Phi\left(\mathbf{e}_{k+1, k+1}\right)=\mathbf{e}_{q-k+1, q-k+1}$, for $k=0,1, \ldots, q-1$.

Proof. Since

$$
\mathbf{e}_{k+1, k+1}=\alpha^{k}\left(\mathbf{e}_{\beta}\right)=V^{-q^{\prime}} g_{1}\left(z^{k p} U\right)+f_{1}\left(z^{k p} U\right)+g_{1}\left(z^{k p} U\right) V^{q^{\prime}},
$$

one has

$$
\Phi\left(\mathbf{e}_{k+1, k+1}\right)=V^{q^{\prime}} g_{1}\left(z^{k p} U^{*}\right)+f_{1}\left(z^{k p} U^{*}\right)+g_{1}\left(z^{k p} U^{*}\right) V^{-q^{\prime}} .
$$

When $\beta>\frac{1}{2 q}$ this is in fact equal to

$$
\mathbf{e}_{r-k, r-k}=V^{-q^{\prime}} g_{1}\left(z^{(r-k-1) p} U\right)+f_{1}\left(z^{(r-k-1) p} U\right)+g_{1}\left(z^{(r-k-1) p} U\right) V^{q^{\prime}},
$$

since

$$
\begin{aligned}
& f_{1}\left(t+\frac{(r-k-1) p}{q}\right)=f_{1}\left(\frac{k p}{q}-t\right), \\
& g_{1}\left(t+\frac{(r-k-1) p}{q}\right)=g_{1}\left(\frac{k p}{q}+\beta-t\right)
\end{aligned}
$$

are easily verified from the construction of $f_{1}$ and $g_{1}$ in case (1) above.

When $\beta<\frac{1}{2 q}$ one sees that (\#) is equal to

$$
\mathbf{e}_{q-k+1, q-k+1}=V^{-q^{\prime}} g_{1}\left(z^{(q-k) p} U\right)+f_{1}\left(z^{(q-k) p} U\right)+g_{1}\left(z^{(q-k) p} U\right) V^{q^{\prime}},
$$

since in this case

$$
f_{1}\left(t+\frac{(q-k) p}{q}\right)=f_{1}\left(\frac{k p}{q}-t\right), \quad g_{1}\left(t+\frac{(q-k) p}{q}\right)=g_{1}\left(\frac{k p}{q}+\beta-t\right)
$$

are easily verified from the construction of $f_{1}$ and $g_{1}$ in case (2).

\subsection{Lemma.}

(i) If $\beta>\frac{1}{2 q}$, then

$$
\begin{aligned}
& \Phi\left(\mathbf{e}_{i+2, i+1}\right)=\alpha^{r-i-2}\left(\mathbf{e}_{21}^{*}\right), \quad \text { for } i=0,1, \ldots, q-2 \text { and } \\
& \Phi\left(\alpha^{q-1}\left(\mathbf{e}_{21}\right)\right)=\alpha^{r-1}\left(\mathbf{e}_{21}^{*}\right) .
\end{aligned}
$$


(ii) If $\beta<\frac{1}{2 q}$, then

$$
\Phi\left(\mathbf{e}_{i+2, i+1}\right)=\alpha^{q-i-1}\left(\mathbf{e}_{21}^{*}\right), \quad \text { for } i=0,1, \ldots, q-2
$$

and

$$
\Phi\left(\alpha^{q-1}\left(\mathbf{e}_{21}\right)\right)=\mathbf{e}_{21}^{*} .
$$

Proof. Begin with the polar decomposition

$$
\mathbf{e}_{22} V \mathbf{e}_{11}=\mathbf{e}_{21}\left|\mathbf{e}_{22} V \mathbf{e}_{11}\right| .
$$

Fix $0 \leqq i \leqq q-1$. Applying $\alpha^{i}$ gives

$$
\mathbf{e}_{i+2, i+2} V \mathbf{e}_{i+1, i+1}=\alpha^{i}\left(\mathbf{e}_{21}\right)\left|\mathbf{e}_{i+2, i+2} V \mathbf{e}_{i+1, i+1}\right| .
$$

The polar decomposition which is adjoint to this is

$$
\mathbf{e}_{i+1, t+1} V^{*} \mathbf{e}_{i+2, i+2}=\alpha^{i}\left(\mathbf{e}_{21}^{*}\right)\left|\mathbf{e}_{i+1, i+1} V^{*} \mathbf{e}_{i+2, i+2}\right| .
$$

Applying $\Phi$ to (1) gives

$$
\Phi\left(\mathbf{e}_{l+2, i+2}\right) V^{*} \Phi\left(\mathbf{e}_{i+1, i+1}\right)=\Phi\left(\alpha^{i}\left(\mathbf{e}_{21}\right)\right)\left|\Phi\left(\mathbf{e}_{i+2, i+2}\right) V^{*} \Phi\left(\mathbf{e}_{i+1, i+1}\right)\right| .
$$

Suppose that $\beta>\frac{1}{2 q}$. Then by Lemma 2.2(i) Eq. (3) becomes

$$
\mathbf{e}_{r-i-1, r-i-1} V^{*} \mathbf{e}_{r-i, r-l}=\Phi\left(\alpha^{i}\left(\mathbf{e}_{21}\right)\right)\left|\mathbf{e}_{r-i-1, r-i-1} V^{*} \mathbf{e}_{r-i, r-i}\right| \text {. }
$$

Applying $\alpha^{r-2 i-2}$ to (2) yields

$$
\mathbf{e}_{r-i-1, r-l-1} V^{*} \mathbf{e}_{r-l, r-l}=\alpha^{r-i-2}\left(\mathbf{e}_{21}^{*}\right)\left|\mathbf{e}_{r-i-1, r-i-1} V^{*} \mathbf{e}_{r-i, r-i}\right|,
$$

and comparison with $\left(3^{\prime}\right)$ using the uniqueness of the polar decomposition, yields $\Phi\left(\alpha^{i}\left(\mathbf{e}_{21}\right)\right)=\alpha^{r-i-2}\left(\mathbf{e}_{21}^{*}\right)$ and so (i) holds.

Now suppose that $\beta<\frac{1}{2 q}$. Then by Lemma 2.2(ii) Eq. (3) becomes

$$
\mathbf{e}_{q-i, q-i} V^{*} \mathbf{e}_{q-i+1, q-i+1}=\Phi\left(\alpha^{i}\left(\mathbf{e}_{21}\right)\right)\left|\mathbf{e}_{q-i, q-i} V^{*} \mathbf{e}_{q-i+1, q-i+1}\right| .
$$

Applying $\alpha^{q-2 i-1}$ to (2) gives

$$
\mathbf{e}_{q-i, q-i} V^{*} \mathbf{e}_{q-i+1, q-i+1}=\alpha^{q-i-1}\left(\mathbf{e}_{21}^{*}\right)\left|\mathbf{e}_{q-i, q-i} V^{*} \mathbf{e}_{q-i+1, q-1+1}\right|,
$$

and, again, comparison with $\left(3^{\prime \prime}\right)$ using the uniqueness of the polar decomposition, yields (ii).

2.4. Theorem. The irrational rotation algebra $\mathrm{A}_{\theta}$ is the inductive limit of an increasing sequence of direct sums of circle algebras which are invariant under the flip automorphism.

Proof. Once it is shown that $C_{q}$ and $D_{q^{\prime}}$ are invariant, then by means of a spectral modification argument carried out in the proof of Theorem 2.1 in Sect. 2 of [2] it will follow that $A_{\theta}$ is the inductive limit of an increasing sequence of direct sums of circle algebras invariant under the flip. It is enough to check that $C_{q}$ is invariant. Lemma 2.2 shows that the tower projections $\mathbf{e}_{k k}$ are mapped unto each other by the flip. Lemma 2.3 shows that $\Phi\left(\mathbf{e}_{l+2, i+1}\right) \in C_{q}$ since $C_{q}$ is $\alpha$-invariant - recall that 
$\alpha=\alpha_{z, 1}^{p}$. Therefore, it follows that $\Phi$ maps the generators $\mathbf{e}_{i j}$ and $v$ back into $C_{q}$ by virtue of equation $(\star \star \star)$.

\section{The AF-Structure of $\mathbf{B}^{\theta}$}

The objective of this section is to apply the preceding results with the two-tower construction to show that $\mathrm{B}^{\theta}$ is an AF-algebra. An outline of the procedure is in order. Since $\mathrm{A}_{\theta}$ can be approximated by the subalgebras $C_{q} \oplus D_{q^{\prime}}$ it follows that $\mathrm{B}^{\theta}$ can be approximated by the subalgebras $C_{q}^{\Phi} \oplus D_{q^{\prime}}^{\Phi}$, where $C_{q}^{\Phi}$ is the fixed point subalgebra of $C_{q}$ under the flip. The main objective now is to show that the subalgebra $C_{q}^{\Phi}$ (and similary also $D_{q^{\prime}}^{\Phi}$ ) is one of the basic builiding blocks considered by $\mathrm{Su}$. Then, as in the proof of Theorem 2.4 above, a modification of the spectral perturbation result in Sect. 2 of [2] can be carried out to show that $\mathrm{B}^{\theta}$ is the inductive limit of an increasing sequence of finite direct sums of Su's basic building blocks.

This last result will have two ramifications. First, it will guarantee that $\mathrm{B}^{\theta}$ has real rank zero. This follows from Theorem 3.0 below which was used by [5, Sect. 9], [4, proof of Theorem 8.1] and [9, Sect. 9.1] as a modification of the result in [1, Theorem 1.3(b), (c)]. Secondly, this result will allow the algebra $\mathrm{B}^{\theta}$ to fall into the classification scheme of $\mathrm{Su}$ ([9, Theorem 8.3] or [10]). And once it falls into that scheme, it will follow from the fact that $K_{1}\left(\mathrm{~B}^{\theta}\right)=0$ (proved by Kumjian in [7]) that $\mathrm{B}^{\theta}$ is an AF-algebra.

Remark. As pointed out to the author by the referee, in order to get a stronger result than Theorem 3.0 (below) one could use Theorems 7.2 and 8.1 of [4]. In fact, this gives another way to get an increasing sequence of direct sums of basic building blocks (in the sense of $\mathrm{Su}$ ) for $\mathrm{B}^{\theta}$ from the approximating subalgebras $C_{q}^{\Phi} \oplus D_{q^{\prime}}^{\Phi}$.

Recall that a basic building block according to $\mathrm{Su}$ [10, Definition 2] is a $\mathrm{C}^{*}$ subalgebra of some $C\left(X, M_{n}\right)$, the continuous functions on a graph $X$ with values in a matrix algebra $M_{n}$, consisting of all functions which have a specific block diagonal form at each vertex of $X$. The block diagonal form can vary with the vertex. Such a basic building block will be referred to as a Su building block.

3.0. Theorem. (cf. [1] \& $\left[10, p\right.$. 224]). If $\mathrm{A}$ is a simple unital $C^{*}$-algebra with a unique tracial state and if $\mathrm{A}$ is the direct limit of a sequence of finite direct sums of Su building blocks, then A has real rank zero. If, in addition, $K_{1}(\mathrm{~A})=0$, then A is an AF-algebra.

Therefore, all that remains is to compute the subalgebra $C_{q}^{\Phi}$ and show that it is a Su building block. For this, two lemmas are needed.

3.1. Lemma. As above let $r$ be the unique positive integer such that $r \equiv 1-q^{\prime}$ $(\bmod q)$ and $1 \leqq r \leqq q$.

(i) If $\beta>\frac{1}{2 q}$, then $\Phi(v)=\alpha^{r-1}\left(v^{*}\right)=\mathbf{e}_{r 1} v^{*} \mathbf{e}_{1 r}$.

(ii) If $\beta<\frac{1}{2 q}$, then $\Phi(v)=v^{*}$.

Proof. Recall that the unitary $v$ is defined by $v=\alpha^{q-1}\left(\mathbf{e}_{21}\right) \cdot \mathbf{e}_{q 1}$ and belongs to $\mathbf{e}_{11} \mathrm{~A}_{\theta} \mathbf{e}_{11}$. Now expand $\mathbf{e}_{q 1}$ as

$$
\mathbf{e}_{q 1}=\mathbf{e}_{q, q-1} \mathbf{e}_{q-1, q-2} \cdots \mathbf{e}_{32} \mathbf{e}_{21},
$$


to get

$$
v=\alpha^{q-1}\left(\mathbf{e}_{21}\right) \mathbf{e}_{q, q-1} \mathbf{e}_{q-1, q-2} \cdots \mathbf{e}_{32} \mathbf{e}_{21} .
$$

If $\beta>\frac{1}{2 q}$, applying $\Phi$ to $(\dagger)$ and using Lemma 2.3(i) gives

$$
\begin{aligned}
\Phi(v) & =\Phi\left(\alpha^{q-1}\left(\mathbf{e}_{21}\right)\right) \Phi\left(\mathbf{e}_{q, q-1}\right) \Phi\left(\mathbf{e}_{q-1, q-2}\right) \cdots \Phi\left(\mathbf{e}_{32}\right) \Phi\left(\mathbf{e}_{21}\right) \\
& =\alpha^{r-1}\left(\mathbf{e}_{21}^{*}\right) \alpha^{r}\left(\mathbf{e}_{21}^{*}\right) \cdots \alpha^{q}\left(\mathbf{e}_{21}^{*}\right) \alpha\left(\mathbf{e}_{21}^{*}\right) \cdots \alpha^{r-3}\left(\mathbf{e}_{21}^{*}\right) \alpha^{r-2}\left(\mathbf{e}_{21}^{*}\right) \\
& =\alpha^{r-1}\left[\mathbf{e}_{21}^{*} \alpha\left(\mathbf{e}_{21}^{*}\right) \cdots \alpha^{q-r+1}\left(\mathbf{e}_{21}^{*}\right) \cdots \alpha^{q-2}\left(\mathbf{e}_{21}^{*}\right) \alpha^{q-1}\left(\mathbf{e}_{21}^{*}\right)\right] \\
& =\alpha^{r-1}\left(v^{*}\right) .
\end{aligned}
$$

To obtain the last equality in (i) one applies $\alpha^{r-1}$ to $(\dagger)$ and gets

$$
\begin{aligned}
\alpha^{r-1}(v)= & \alpha^{r+q-2}\left(\mathbf{e}_{21}\right) \alpha^{r+q-3}\left(\mathbf{e}_{21}\right) \ldots \alpha\left(\mathbf{e}_{21}\right) \mathbf{e}_{21} \alpha^{q-1}\left(\mathbf{e}_{21}\right) \alpha^{q-2}\left(\mathbf{e}_{21}\right) \\
& \cdots \alpha^{r}\left(\mathbf{e}_{21}\right) \alpha^{r-1}\left(\mathbf{e}_{21}\right) \mathbf{e}_{r r} \\
= & \alpha^{r+q-2}\left(\mathbf{e}_{21}\right) \alpha^{r+q-3}\left(\mathbf{e}_{21}\right) \cdots \alpha\left(\mathbf{e}_{21}\right) \mathbf{e}_{21} v \mathbf{e}_{1 q} \alpha^{q-2}\left(\mathbf{e}_{21}\right) \\
& \cdots \alpha^{r}\left(\mathbf{e}_{21}\right) \alpha^{r-1}\left(\mathbf{e}_{21}\right) \mathbf{e}_{r, r-1} \mathbf{e}_{r-1, r-2} \ldots \mathbf{e}_{21} \mathbf{e}_{12} \mathbf{e}_{23} \cdots \mathbf{e}_{r-1, r} \\
= & \mathbf{e}_{r+q, r+q-1} \mathbf{e}_{r+q-1, r+q-2} \ldots \mathbf{e}_{32} \mathbf{e}_{21} v \mathbf{e}_{1 q} \mathbf{e}_{q, q-1} \\
& \cdots \mathbf{e}_{r+2, r+1} \mathbf{e}_{r+1, r} \mathbf{e}_{r, r-1} \mathbf{e}_{r-1, r-2} \ldots \mathbf{e}_{21} \mathbf{e}_{12} \mathbf{e}_{23} \cdots \mathbf{e}_{r-1, r} \\
= & \mathbf{e}_{r 1} v \mathbf{e}_{1 r} .
\end{aligned}
$$

If $\beta<\frac{1}{2 q}$, applying $\Phi$ to ( $\dagger$ ) and using Lemma 2.3(ii) yields

$$
\begin{aligned}
\Phi(v) & =\Phi\left(\alpha^{q-1}\left(\mathbf{e}_{21}\right)\right) \Phi\left(\mathbf{e}_{q, q-1}\right) \Phi\left(\mathbf{e}_{q-1, q-2}\right) \cdots \Phi\left(\mathbf{e}_{32}\right) \Phi\left(\mathbf{e}_{21}\right) \\
& =\mathbf{e}_{21}^{*} \alpha\left(\mathbf{e}_{21}^{*}\right) \alpha^{2}\left(\mathbf{e}_{21}^{*}\right) \cdots \alpha^{q-2}\left(\mathbf{e}_{21}^{*}\right) \alpha^{q-1}\left(\mathbf{e}_{21}^{*}\right) \\
& =v^{*}
\end{aligned}
$$

as required.

\subsection{Lemma.}

(i) If $\beta>\frac{1}{2 q}$, then

$$
\Phi\left(\mathbf{e}_{i 1}\right)= \begin{cases}\mathbf{e}_{r-\imath+1, r} & \text { for } i \leqq r \\ \mathbf{e}_{r-i+1,1} v^{*} \mathbf{e}_{1 r} & \text { for } i>r\end{cases}
$$

for $i=1,2, \ldots, q$.

(ii) If $\beta<\frac{1}{2 q}$, then

$$
\Phi\left(\mathbf{e}_{i 1}\right)= \begin{cases}\mathbf{e}_{11} & \text { for } i=1 \\ \mathbf{e}_{q-i+2,1} v^{*} & \text { for } i \geqq 2\end{cases}
$$

for $i=1,2, \ldots, q$.

Proof. When $i=1$ this follows from Lemma 2.2. Otherwise, the relations follow by applying Lemma 2.3 to the expansion $\mathbf{e}_{l 1}=\mathbf{e}_{i, t-1} \mathbf{e}_{i-1, i-2} \ldots \mathbf{e}_{32} \mathbf{e}_{21}$. 
Recall that $C_{q}$ is isomorphic to $M_{q}(C(\mathbb{T}))$ and is generated by the matrix units $\left(\mathbf{e}_{i j}\right)$ and partial isometry $v$ having the projection $\mathbf{e}_{11}$ as range and support. So its elements have the form

$$
X=\sum_{i, j=1}^{q} \mathbf{e}_{i 1} x^{l j}(v) \mathbf{e}_{1 j},
$$

where $x^{i j} \in C(\mathbb{T})$. This element is, in effect, the $q \times q$ matrix $\left[x^{i j}\right]$ of $M_{q}(C(\mathbb{T}))$. So $X$ will be identified with this matrix form when convenient.

First, suppose that $\beta>\frac{1}{2 q}$. Then in view of Lemmas 3.1(i) and 3.2(i) the following summation can be split as

$$
\begin{aligned}
\Phi(X)= & \sum_{i, j=1}^{q} \Phi\left(\mathbf{e}_{i 1}\right) x^{i j}(\Phi(v)) \Phi\left(\mathbf{e}_{1 j}\right) \\
= & \sum_{i, j=1}^{q} \Phi\left(\mathbf{e}_{i 1}\right) \mathbf{e}_{r 1} x^{i j}\left(v^{*}\right) \mathbf{e}_{1 r} \Phi\left(\mathbf{e}_{1 j}\right) \\
= & \sum_{i, j=1}^{r} \mathbf{e}_{r-i+1,1} x^{i j}\left(v^{*}\right) \mathbf{e}_{1, r-j+1}+\sum_{i=1}^{r} \sum_{j=r+1}^{q} \mathbf{e}_{r-l+1,1} x^{i j}\left(v^{*}\right) v \mathbf{e}_{1, r-j+1} \\
& +\sum_{l=r+1}^{q} \sum_{j=1}^{r} \mathbf{e}_{r-i+1,1} v^{*} x^{i j}\left(v^{*}\right) \mathbf{e}_{1, r-j+1}+\sum_{i, j=r+1}^{q} \mathbf{e}_{r-i+1,1} x^{i j}\left(v^{*}\right) \mathbf{e}_{1, r-j+1} .
\end{aligned}
$$

Now upon replacing $i$ by $r+1-i$ and $j$ by $r+1-j$ in each of the last four summations they become (respectively)

$$
\begin{aligned}
\Phi(X)= & \sum_{i, j=1}^{r} \mathbf{e}_{i 1} x^{r+1-i, r+1-j}\left(v^{*}\right) \mathbf{e}_{1 j}+\sum_{i=1}^{r} \sum_{j=r+1}^{q} \mathbf{e}_{i 1} x^{r+1-l, r+1-j}\left(v^{*}\right) v \mathbf{e}_{1_{j}} \\
& +\sum_{i=r+1}^{q} \sum_{j=1}^{r} \mathbf{e}_{i 1} v^{*} x^{r+1-l, r+1-j}\left(v^{*}\right) \mathbf{e}_{1 j}+\sum_{i, j=r+1}^{q} \mathbf{e}_{l 1} x^{r+1-i, r+1-j}\left(v^{*}\right) \mathbf{e}_{1 j} .
\end{aligned}
$$

Writing out the matrix form for this element one obtains

$$
\left[\begin{array}{ccc|cccc}
x^{r r}\left(v^{*}\right) & \cdots & x^{r 1}\left(v^{*}\right) & x^{r q}\left(v^{*}\right) v & x^{r, q-1}\left(v^{*}\right) v & \cdots & x^{r, r+1}\left(v^{*}\right) v \\
\vdots & \ddots & \vdots & \vdots & \vdots & \ddots & \vdots \\
x^{1 r}\left(v^{*}\right) & \cdots & x^{11}\left(v^{*}\right) & x^{1 q}\left(v^{*}\right) v & x^{1, q-1}\left(v^{*}\right) v & \cdots & x^{1, r+1}\left(v^{*}\right) v \\
\hline v^{*} x^{q r}\left(v^{*}\right) & \cdots & v^{*} x^{q 1}\left(v^{*}\right) & x^{q q}\left(v^{*}\right) & x^{q, q-1}\left(v^{*}\right) & \cdots & x^{q, r+1}\left(v^{*}\right) \\
v^{*} x^{q-1, r}\left(v^{*}\right) & \cdots & v^{*} x^{q-1,1}\left(v^{*}\right) & x^{q-1, q}\left(v^{*}\right) & x^{q-1, q-1}\left(v^{*}\right) & \cdots & x^{q-1, r+1}\left(v^{*}\right) \\
\vdots & \ddots & \vdots & \vdots & \vdots & \ddots & \vdots \\
v^{*} x^{r+1, r}\left(v^{*}\right) & \cdots & v^{*} x^{r+1,1}\left(v^{*}\right) & x^{r+1, q}\left(v^{*}\right) & x^{r+1, q-1}\left(v^{*}\right) & \cdots & x^{r+1, r+1}\left(v^{*}\right)
\end{array}\right]
$$

and a moment's reflection will show that this matrix is equal to

$$
Z\left[x^{i j}\left(v^{*}\right)\right] Z^{*},
$$

where $Z$ is the $q \times q$ block matrix

$$
Z=\left[\begin{array}{cc}
S_{1} & \mathbf{0} \\
\mathbf{0} & v^{*} S_{2}
\end{array}\right]
$$


and $S_{1}$ and $S_{2}$ are the $r \times r$ and $(q-r) \times(q-r)$ matrices, respectively, of the form

$$
\left[\begin{array}{lllll}
0 & 0 & \cdots & 0 & 1 \\
0 & 0 & \cdots & 1 & 0 \\
0 & 0 & \therefore & 0 & 0 \\
0 & 1 & \cdots & 0 & 0 \\
1 & 0 & \cdots & 0 & 0
\end{array}\right]
$$

which flips the rows of a matrix when pre-multiplied and flips its columns when post-multiplied. Hence,

$$
\Phi\left(\left[x^{i j}(v)\right]\right)=Z\left[\left(x^{i j}\left(v^{*}\right)\right] Z^{*},\right.
$$

giving the explicit form of the flip on $C_{q}$. Now if $X \in C_{q}^{\Phi}$ then in terms of functions $X \in C\left([0,1], M_{q}\right)$ of the variable $t$ this equation entails

$$
X(t)=Z_{t} X(1-t) Z_{t}^{*} .
$$

So, in effect, $X$ is a function defined on the interval $\left[0, \frac{1}{2}\right]$ where at its end points $X(0)$ commutes with the self-adjoint unitary $Z_{0}=\left[\begin{array}{cc}S_{1} & \mathbf{0} \\ \mathbf{0} & S_{2}\end{array}\right]$ and $X\left(\frac{1}{2}\right)$ commutes with the self-adjoint unitary $Z_{\frac{1}{2}}=\left[\begin{array}{cc}S_{1} & \mathbf{0} \\ \mathbf{0} & -S_{2}\end{array}\right]$. This means that $C_{q}^{\Phi}$ is isomorphic to the subalgebra of the continuous functions in $C\left(\left[0, \frac{1}{2}\right], M_{q}\right)$ which have block diagonal form at the end points. Therefore, $C_{q}^{\Phi}$ is a $\mathrm{Su}$ building block.

Finally, consider the case $\beta<\frac{1}{2 q}$. By virtue of Lemmas 3.1(ii) and 3.2(ii) the summation for $\Phi(X)$ is

$$
\begin{aligned}
\Phi(X)= & \sum_{i, j=1}^{q} \Phi\left(\mathbf{e}_{i 1}\right) x^{i j}\left(v^{*}\right) \Phi\left(\mathbf{e}_{1 j}\right) \\
= & \mathbf{e}_{11} x^{11}\left(v^{*}\right) \mathbf{e}_{11}+\sum_{j=2}^{q} \mathbf{e}_{11} x^{1 j}\left(v^{*}\right) v \mathbf{e}_{1, q-j+2} \\
& +\sum_{i=2}^{q} \mathbf{e}_{q-i+2,1} v^{*} x^{i 1}\left(v^{*}\right) \mathbf{e}_{11}+\sum_{l, j=2}^{q} \mathbf{e}_{q-i+2,1} x^{i j}\left(v^{*}\right) \mathbf{e}_{1, q-j+2} .
\end{aligned}
$$

Upon replacing $i$ by $q+2-i$ and $j$ by $q+2-j$ in each of the last three summations they become (respectively)

$$
\begin{aligned}
\Phi(X) & =\mathbf{e}_{11} x^{11}\left(v^{*}\right) \mathbf{e}_{11}+\sum_{j=2}^{q} \mathbf{e}_{11} x^{1, q-j+2}\left(v^{*}\right) v \mathbf{e}_{1 j} \\
& +\sum_{i=2}^{q} \mathbf{e}_{i 1} v^{*} x^{q-i+2,1}\left(v^{*}\right) \mathbf{e}_{11}+\sum_{i, j=2}^{q} \mathbf{e}_{i 1} x^{q-i+2, q-j+2}\left(v^{*}\right) \mathbf{e}_{1 j} .
\end{aligned}
$$

This is, in effect, the $q \times q$ matrix

$\left[\begin{array}{c|cccc}x^{11}\left(v^{*}\right) & x^{1 q}\left(v^{*}\right) v & x^{1, q-1}\left(v^{*}\right) v & \cdots & x^{12}\left(v^{*}\right) v \\ \hline v^{*} x^{q 1}\left(v^{*}\right) & x^{q q}\left(v^{*}\right) & x^{q, q-1}\left(v^{*}\right) & \cdots & x^{q 2}\left(v^{*}\right) \\ v^{*} x^{q-1,1}\left(v^{*}\right) & x^{q-1, q}\left(v^{*}\right) & x^{q-1, q-1}\left(v^{*}\right) & \cdots & x^{q-1,2}\left(v^{*}\right) \\ \vdots & \vdots & \vdots & \ddots & \vdots \\ v^{*} x^{21}\left(v^{*}\right) & x^{2 q}\left(v^{*}\right) & x^{2, q-1}\left(v^{*}\right) & \cdots & x^{22}\left(v^{*}\right)\end{array}\right]$,


which is easily seen to be

$$
Z\left[x^{i j}\left(v^{*}\right)\right] Z^{*},
$$

where $Z$ is the $q \times q$ matrix

$$
\left[\begin{array}{cccccc}
v & 0 & 0 & \cdots & 0 & 0 \\
0 & 0 & 0 & \cdots & 0 & 1 \\
0 & 0 & 0 & \cdots & 1 & 0 \\
0 & 0 & 0 & \cdots & 0 & 0 \\
0 & 0 & 1 & \cdots & 0 & 0 \\
0 & 1 & 0 & \cdots & 0 & 0
\end{array}\right] .
$$

Consequently,

$$
\Phi\left(\left[x^{i j}(v)\right]\right)=Z\left[x^{i j}\left(v^{*}\right)\right] Z^{*} .
$$

Therefore, if $X \in C_{q}^{\Phi}$ then (as a function of the variable $t$ ) this equation entails

$$
X(t)=Z_{t} X(1-t) Z_{t}^{*}
$$

which, as before, means that $X$ is a function defined on the interval $\left[0, \frac{1}{2}\right]$ and has block diagonal form at the end points. Therefore, $C_{q}^{\Phi}$ is again a $\mathrm{Su}$ building block.

\section{Appendix}

Since $\mathrm{B}^{\theta}$ is strongly Morita equivalent to the crossed product $\mathrm{B}_{\theta}=\mathrm{A}_{\theta} \times_{\Phi} \mathbb{Z}_{2}$ (and both being unital $\mathrm{C}^{*}$-algebras), if it can be shown that $\mathrm{B}_{\theta}$ has a unique tracial state, then the same will hold for $\mathrm{B}^{\theta}$, in view of Rieffel's Proposition 2.2 of [8].

Let $W$ denote the canonical unitary implementing the flip automorphism so that the algebra $\mathrm{B}_{\theta}$ is generated by 3 unitaries $U, V, W$ satisfying

$$
\begin{aligned}
V U & =\lambda U V, \\
W U W & =U^{*}, \\
W V W & =V^{*}, \\
W^{2} & =1 .
\end{aligned}
$$

Proposition. [3, Theorem 4.5] If $\theta$ is irrational, then the $C^{*}$-algebra $\mathrm{B}_{\theta}$ has a unique tracial state.

Proof. Let $\tau$ be any tracial state on $\mathrm{B}_{\theta}$. It restricts to the unique one on $\mathrm{A}_{\theta}$. Since the unitaries $U^{p} V^{q}$ and $U^{p} V^{q} W$ are total in $\mathrm{B}_{\theta}$, it is sufficient to show that $\tau\left(U^{p} V^{q} W\right)=0$ for all integers $p, q$. So fix $p, q$ and note that for all integers $k$

$$
\begin{aligned}
\tau\left(U^{p} V^{q} W\right) & =\tau\left(U^{k} U^{p} V^{q} W U^{-k}\right) \\
& =\tau\left(U^{p+k} V^{q} U^{k} W\right) \\
& =\lambda^{q k} \tau\left(U^{p+2 k} V^{q} W\right) .
\end{aligned}
$$


For each $n \in \mathbb{N}$, consider the element

$$
\begin{aligned}
x_{n} & =\frac{1}{n+1}\left(U^{p} V^{q} W+\lambda^{q} U^{p+2} V^{q} W+\cdots+\lambda^{n q} U^{p+2 n} V^{q} W\right) \\
& =\frac{1}{n+1}\left(1+\lambda^{q} U^{2}+\cdots+\lambda^{n q} U^{2 n}\right) U^{p} V^{q} W .
\end{aligned}
$$

From $\left({ }^{*}\right)$ it follows that $\tau\left(x_{n}\right)=\tau\left(U^{p} V^{q} W\right)$ for all $n$. The product

$$
x_{n} x_{n}^{*}=\frac{1}{(n+1)^{2}} \sum_{i, j=0}^{n} \lambda^{q(i-j)} U^{2(i-j)}
$$

has trace

$$
\begin{aligned}
\tau\left(x_{n} x_{n}^{*}\right) & =\frac{1}{(n+1)^{2}} \sum_{i, j=0}^{n} \lambda^{q(i-j)} \tau\left(U^{2(i-j)}\right) \\
& =\frac{1}{(n+1)^{2}} \sum_{i=0}^{n} \tau(1) \\
& =\frac{1}{n+1} .
\end{aligned}
$$

The continuity of $\tau$ and its associated Schwartz inequality yield

$$
\left|\tau\left(U^{p} V^{q} W\right)\right|^{2}=\left|\tau\left(x_{n}\right)\right|^{2} \leqq \tau\left(x_{n} x_{n}^{*}\right) \cdot \tau(1)=\frac{1}{n+1},
$$

and since $n$ was arbitrary, it follows that $\tau\left(U^{p} V^{q} W\right)=0$.

Acknowledgements. The author is highly indebted to George Elliott for his unabated encouragement and for suggesting the problem considered in this paper. He is very thankful to the referee for pointing out a gap in the original proof of Theorem 2.4 and for suggesting that it can be bridged using Theorem 2.1 of [2]. The referee made many other valuable suggestions to an earlier version of the present paper. The author wishes to thank Ola Bratteli for providing him with his papers [2] and [4], and to Hongbing Su for providing him with a preprint of [9]. He also wishes to thank the Mathematics Department at the University of Western Ontario for their warm hospitality and support while this research was done. The author is indebted to NSERC for supporting this research.

Note added in Proof. Since the completion of this paper the author has learned that Elliott and Q. Lin had obtained another proof that $\mathrm{A}_{\theta}$ is the inductive limit of direct sums of basic building blocks consisting of type I C*-algebras ("Cut-down method in the inductive limit decomposition of non-commutative tori," to appear in J. London Math. Soc.). Subsequently, Florin Boca has shown that these building blocks are in fact flip invariant and obtained yet another proof of the result of Bratteli and Kishimoto ("The structure of the flip fixed point algebra in certain noncommutative 3-tori," preprint).

\section{References}

1. Blackadar, B., Bratteli, O., Elliott, G., Kumjian, A.: Reduction of real rank in inductive limits of $\mathrm{C}^{*}$-algebras. Math. Ann. 292, 111-126 (1992)

2. Bratteli, O., Evans, D., Kishimoto, A.: Crossed products of totally disconnected spaces by $\mathbb{Z}_{2} * \mathbb{Z}_{2}$. Ergod. Th. Dynam. Sys. 13, 445-484 (1993) 
3. Bratteli, O., Elliott, G., Evans, D., Kishimoto, A.: Non-commutative spheres I. Int. J. Math. 2, no. 2, 139-166 (1991)

4. Bratteli, O., Kishimoto A.: Non-commutative spheres III. Irrational Rotations. Commun. Math. Phys. 147, 605-624 (1992)

5. Elliott, G.: A classification of certain simple $C^{*}$-algebras. Quantum and Non-Commutative Analysis, Araki et al. (eds.), Kluwer, 1993

6. Elliott, G., Evans, D.: The structure of the irrational rotation $\mathrm{C}^{*}$-algebra. Ann. Math. 138, 477-501 (1993)

7. Kumjian, A.: On the $K$-theory of the symmetrized non-commutative torus. C. R. Math. Rep. Acad. Sci. Canada XII, no. 3, 87-89 (1990)

8. Rieffel, M.: $C^{*}$-algebras associated with irrational rotations. Pac. J. Math. 93, no. 2, 415-429 (1981)

9. $\mathrm{Su}, \mathrm{H}$.: On the classification of $\mathrm{C}^{*}$-algebras of real rank zero: Inductive limits of matrix algebras over non-Hausdorff graphs. Memoirs A.M.S. (to appear); Ph.D. Thesis, Unıversity of Toronto (1992)

10. $\mathrm{Su}, \mathrm{H}$ :: On the classification of $\mathrm{C}^{*}$-algebras of real rank zero: Inductive limits of matrix algebras over graphs. C. R. Math. Rep. Acad. Sci. Canada 13, 223-228 (1991)

11. Voiculescu, D.: Almost inductive limit automorphisms and embeddings into AF-algebras. Ergod. Th. Dynam. Sys. 6 (1986)

12. Walters, S.: Projective modules over the non-commutative sphere. J. London Math. Soc. (to appear)

Communicated by A. Connes 
